\title{
Estudo da posição natural da cabeça em relação ao plano horizontal de Frankfurt na avaliação mandibular de indivíduos com padrão facial de Classe I e Classe II*
}

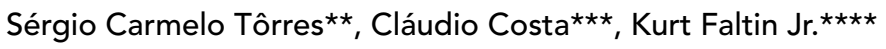

\section{Resumo}

Objetivo: avaliar o comportamento do Plano de Frankfurt e da leitura de posição mandibular em indivíduos com padrões faciais de Classe I e II, relacionados à postura natural da cabeça. Metodologia: fotografias do perfil em PNC foram obtidas dos pacientes em pé e relaxados, olhando a imagem de seus próprios olhos refletida em um espelho colocado $1 \mathrm{~m}$ à frente deles. Um prumo foi utilizado para definir a linha vertical (VER) nas fotografias e uma linha passando pela glabela e pogônio mole foi transferida das fotografias para as telerradiografias laterais. Uma linha horizontal (HOR), perpendicular à linha vertical, comparada com a referência intracraniana plano horizontal de Frankfurt (HF), foi utilizada para avaliação da variação deste plano em posição natural da cabeça. Foram avaliadas as alterações da PNC sobre a medida cefalométrica da posição sagital do contorno anterior da mandíbula pela diferença entre Nperp-P (McNamara) e Nvert-P. Analisou-se os registros fotográficos e radiográficos pré-tratamento de 60 pacientes selecionados em dois grupos baseados no padrão esquelético facial de Classe I e Classe II. Resultados e Conclusões: os resultados obtidos demonstraram uma alta variação interindividual entre o plano horizontal de Frankfurt (HF) e a linha horizontal verdadeira (HOR) em ambos grupos. Porém, a diferença média apresentou-se mínima entre estas referências, estatisticamente não significante e semelhante nos dois grupos. A relação observada da variação do ângulo HOR.HF para o cálculo estimado da diferença entre Nperp$\mathrm{P}$ e Nvert-P retornou um coeficiente de 1: 2,119 \pm 0,029. Esta discrepância do diagnóstico sagital mandibular compromete o planejamento ortodôntico nos pacientes que apresentaram diferença entre os planos horizontais intra e extra-cranianos. Destacamos a importância das avaliações faciais e cefalométricas em norma lateral serem realizadas em posição natural da cabeça, utilizando as linhas horizontal e vertical verdadeiras como referências.

Palavras-chave: Posição natural da cabeça. Cefalometria. Diagnóstico.

* Resumo da dissertação apresentada ao Programa de Pós-Graduação em Odontologia da Universidade Paulista - UNIP, área de concentração em Clínica Infantil - Ortodontia, para a obtenção do título de Mestre.

** Especialista em Ortodontia pela PROFIS - USP - Bauru. Mestre em Ortodontia pela Universidade Paulista - SP. Professor do Curso de Aperfeiçoamento em Ortodontia da ABCD-MG

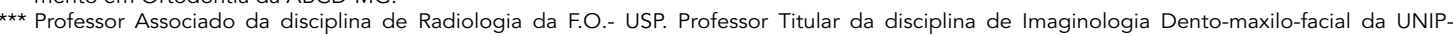
São Paulo e UNIP- Campinas. Professor Titular da disciplina de Diagnóstico por Imagem em Odontologia da UNICSUL.

**** Professor Titular da disciplina de Ortodontia da UNIP. Coordenador dos cursos de Pós-graduação da UNIP- São Paulo. Pós-graduado em Ortopedia Maxilar pela Universidade de Bonn- Alemanha. 


\section{INTRODUÇÃO}

A posição natural da cabeça (PNC) tem sido apresentada nos estudos antropométricos e ortodônticos como referência para avaliação da morfologia craniofacial $1^{4,5,14,15,22,38}$.

Os métodos de obtenção da PNC apresentam algumas variações e controvérsias. A visualização das pupilas num espelho e a utilização de uma corrente metálica unida a um prumo posicionada à margem anterior do chassi porta-filme, possível de ser visualizada à frente do contorno do perfil facial na telerradiografia lateral, compoem o método mais empregado $2,4,8,9,10,14,15,21,23,26,27,35,37,38,41,50,53,60,61,66,67,68$. Esta linha extra-craniana foi denominada vertical verdadeira (VER). A partir desta referência vertical, pode-se traçar uma perpendicular, obtendo-se a linha denominada horizontal verdadeira (HOR). As olivas do cefalostato introduzidas nos meatos acústicos alterariam a posição da cabeça ${ }^{12,20,57,67,68}$. $\mathrm{O}$ método fotográfico para registrar a PNC emprega fotografias dos pacientes em pé e relaxados, olhando para suas pupilas no espelho localizado a 1 metro de distância à sua frente. Utiliza-se uma linha de chumbo para definir a linha vertical verdadeira (VER) nas fotografias. Uma linha horizontal (HOR), perpendicular à vertical é traçada, assim como uma linha do perfil mole unindo os pontos Násio e Pogônio (N'- Pog') do paciente. As linhas HOR e VER são transferidas da fotografia para a telerradiografia, através dos ângulos entre a linha N'- Pog' e as linhas HOR e VER ${ }^{26,28 .}$

Esta posição da cabeça com a qual o paciente se conduz em seu dia a dia, reproduz mais fielmente o seu perfil facial. O clínico, portanto, deveria utilizá-la, especialmente durante o tratamento ortopédico ou ortodôntico-cirúrgico das deformidades dentofaciais ${ }^{4,10,14,15,35,37,38,53,66}$.

Nos últimos cinqüenta anos a posição natural da cabeça tem sido adotada como a postura correta natural do corpo e o alinhamento com a coluna cervical, determinada pelo equilíbrio da cabeça e do corpo quando o indivíduo olha para frente $^{67,68}$. A reprodutibilidade da PNC em inter- valos distintos tem sido considerada clinicamente aceitável dentro de um espectro de variação de até $4^{\circ}$, certamente muito melhor do que os $26^{\circ}$ de variabilidade do plano horizontal de Frankfurt e a linha Sela-Násio entre os indivíduos ${ }^{12,34,40,66,71}$. Estas referências intracranianas utilizadas pelas análises cefalométricas de Steiner, Ricketts, Schwarz, McNamara, entre outras, apresentam diagnósticos completamente diferentes para um mesmo paciente, resultando em um planejamento ortodôntico-ortopédico equivocado $32,47,48,52,54,55,65$. As maiores vantagens da orientação da PNC seriam a obtenção de um plano de referência horizontal com menor variação ao longo do tempo e um grau de reprodutibilidade mais confiável que as referências cefalométricas intracranianas $^{4,9,12,18,23,26,29,38,41,42,45,49,50,53,60,65,68}$, possibilitando a utilização de linhas extra-cranianas na avaliação cefalométrica e também verificar a confiabilidade das linhas de referência intracranianas.

Estudos longitudinais da reprodutibilidade da PNC foram realizados por Cooke ${ }^{11}$, Peng e Cooke $^{41}$, abrangendo períodos de 5 e 15 anos, respectivamente, em 20 pacientes com idade inicial média de 12 anos. Eles obtiveram uma variação média da PNC de $1,9^{\circ}$ após 5 a 10 minutos do primeiro registro; de $2,3^{\circ}$ após 3 a 6 meses; de $3^{\circ}$ após 5 anos e de apenas $2,2^{\circ}$ após 15 anos. A reprodutibilidade ultrapassou o limite do tempo, tornando-se dessa forma confiável a longo prazo.

Uma referência vertical através do ponto Násio foi inicialmente apresentada por McNamara $\mathrm{Jr}^{33}$, utilizando uma perpendicular a Frankfurt (Nperp). Porém, a utilização de uma perpendicular do Násio com a horizontal verdadeira (HOR), na avaliação da relação ântero-posterior da maxila e da mandíbula com medidas lineares, tem sido proposta por alguns autores $12,23,26,43,44,65,68$.

A tendência da postura da cabeça camuflar a sua morfologia, citada por alguns trabalhos na literatura, associada com os resultados de estudos em respiradores bucais, indicando uma extensão da posição da cabeça, devem ser analisados e considera- 
dos na avaliação do paciente $3,6,17,25,39,56,58,59,60,61,69,70$.

A análise facial tem sido essencial no diagnóstico e planejamento ortodônticos, por expressar a real estética do paciente, visualizada em norma lateral e frontal para a avaliação das alterações faciais associadas com compensações dos tecidos moles. Nesta análise, muitas vezes nos defrontamos com discrepâncias entre os resultados cefalométricos e os resultados expressos no perfil facial. Logicamente, deveríamos priorizar a correção das alterações visualizadas neste contorno do perfil mole do paciente, obtendo-se a correção ortodôntica associada com possiveis melhorias estéticas faciais ${ }^{1,12}$. Neste contexto, a posição da cabeça exerce um fator importante na avaliação, pois claramente os desvios de inclinação natural alteram, principalmente, a posição sagital da mandíbula e do mento em relação à vertical verdadeira, influenciando o planejamento ortodôntico, ortopédico e cirúrgico.

Portanto, a correta avaliação da posição natural da cabeça, sua utilização como método prioritário de diagnóstico e sua influência sobre a morfologia facial devem ser avaliados corretamente, para uma elaboração de um protocolo de análises cefalométrica e facial adequadas nesta postura.

\section{PROPOSIÇÃO}

O objetivo deste trabalho foi avaliar fotografias obtidas em posição natural da cabeça e telerradiografias em norma lateral de 60 indivíduos, quanto aos seguintes aspectos:

1) A posição natural da cabeça nos padrões faciais de Classe I e Classe II, por meio da diferença entre a linha horizontal verdadeira e o plano horizontal de Frankfurt.

2) A influência da PNC sobre a posição sagital da mandíbula, comparando a diferença do valor da distância linear entre as grandezas Nperp-P (McNamara) e Nvert-P.

\section{MATERIAL E MÉTODOS}

\section{A amostra}

A amostra foi composta por indivíduos leuco- dermas brasileiros, sem tratamento ortodôntico e/ou ortopédico prévios, respiradores nasais, divididos em 2 grupos, conforme a relação das posições ântero-posteriores das bases ósseas, segundo Martins ${ }^{31}$ :

- Indivíduos com perfil facial harmonioso e padrão esquelético de Classe I, apresentando o valor do ângulo ANB (Steiner) $=4^{\circ} \pm 2^{\circ}$ (dentadura mista) ou $2^{\circ} \pm 2^{\circ}$ (maturidade esquelética);

- Indivíduos com perfil facial muito convexo e padrão esquelético de Classe II, apresentando o ângulo ANB (Steiner) maior que $6^{\circ}$ (dentadura mista) ou maior que $4^{\circ}$ (maturidade esquelética) e retrusão mandibular verificada pelo ângulo SNB menor que $74^{\circ}$ (dentadura mista) ou menor que $76,5^{\circ}$ (maturidade esquelética).

$\mathrm{O}$ protocolo de pesquisa com informações e consentimento dos registros fotográficos e radiográficos dos indivíduos foi aprovado pelo Conselho de Ética da Universidade Paulista, sendo estabelecido um número para cada paciente, preservando totalmente a sua identidade. As radiografias foram tomadas com a finalidade de diagnóstico ortodôntico e não somente para a pesquisa realizada.

\section{Método de registro da posição natural da cabeça}

A posição natural da cabeça (PNC) foi obtida com os indivíduos em pé, relaxados, com os pés afastados aproximadamente $10 \mathrm{~cm}$, inclinando a cabeça para frente e para trás, diminuindo a amplitude até sentir que foi alcançado o seu equilíbrio natural.

Um espelho oval colocado 1 metro à frente do paciente, foi utilizado como dispositivo visual, orientando-o para observar seus próprios olhos refletidos, mantendo as pupilas no centro ocular Torna-se importante lembrar que o espelho não possuía bordas retas, pois o paciente naturalmente poderia guiar-se por essas referências.

Quando a PNC foi obtida e verificada a posição das pupilas no centro ocular, uma fotografia lateral foi realizada, sempre pelo mesmo operador.

Ressaltamos que o método fotográfico para 
registro da PNC foi utilizado por consideramos esta uma adaptação mais prática e simples para obtenção do registro da PNC, sendo facilmente transferivel para todas as telerradiografias laterais. Verificamos também que a PNC é mais precisa no registro fotográfico, pois ao contrário do que possa ocorrer durante o posicionamento do paciente no cefalostato, durante a tomada radiográfica, temos uma posição mais relaxada e com menores influências na obtenção do registro fotográfico. Outra vantagem é a fidelidade na análise do perfil facial para diagnóstico e planejamento, utilizandose a fotografia da cabeça posicionada na inclinação em que o paciente se conduz no seu dia a dia, expressando a real estética facial na avaliação das alterações e compensações faciais.

\section{Método para obtenção das fotografias laterais}

Após a orientação para a obtenção da PNC, uma linha vertical verdadeira foi registrada através de uma corrente metálica unida a um chumbo, posicionada próxima ao perfil mole do indivíduo, de maneira que sua imagem apareça no registro fotográfico.

O indivíduo foi orientado também para manter os dentes em oclusão e a musculatura peribucal relaxada.

Para a tomada fotográfica, utilizamos uma câmera Pentax ${ }^{\circledR}$ K-1000 com lente objetiva macro $105 \mathrm{~mm}$ Vivitar $^{\circledR}$ e flash circular Sunpak ${ }^{\circledR}$. A câmera foi montada verticalmente num tripé a $1,5 \mathrm{~m}$, lateralmente, da face do paciente, exatamente perpendicular ao perfil facial e centralizando o enquadramento nos tecidos moles faciais.

Em seguida, verificamos se a linha metálica à frente do contorno facial estava incluída na imagem a ser registrada. Ao nos certificarmos da PNC, realizamos a tomada fotográfica do indivíduo.

\section{Método de obtenção das telerradiografias em norma lateral}

Os indivíduos foram examinados através de telerradiografias em norma lateral da cabeça, sempre pelo mesmo operador, sendo a distância foco-filme de 1,56m. Empregamos o aparelho de raios $\mathrm{X}$ da marca Siemens ${ }^{\circledR}$, com regime de trabalho de $70 \mathrm{kVp}$ e $10 \mathrm{~mA}$ e tempo de exposição de $1,2 \mathrm{~s}$, com a linha mediana do cefalostato a $1,52 \mathrm{~m}$ de distância da fonte de raios X. Os filmes radiográficos utilizados foram da marca Kodak ${ }^{\circledR}$, com dimensões de $18 \times 24 \mathrm{~cm}$, com seu longo eixo no sentido vertical, com a utilização de placas intensificadoras da marca Kodak ${ }^{\circledR}$, tipo X-Omatic Lanex com velocidade regular e filtro de alumínio chanfrado justaposto ao chassi porta-filme para evidenciar o tecido mole do perfil facial, sendo os filmes processados através de processadora automática da marca Gendex-GPX ${ }^{\circledR}$ modelo 110-0096G-1, com soluções químicas da marca Kodak ${ }^{\circledR}$.

Os indivíduos deveriam estar em posição de oclusão e a musculatura peribucal relaxada.

\section{Técnica para obtenção dos traçados}

Nas fotografias laterais (Fig. 1)

As linhas de referências das fotografias laterais foram realizadas utilizando folhas de acetato U1traphan ${ }^{\circledR}$ para traçado cefalométrico de $0,075 \mathrm{~mm}$ de espessura e lapiseira com grafite preto de diâmetro $0,5 \mathrm{~mm}$.

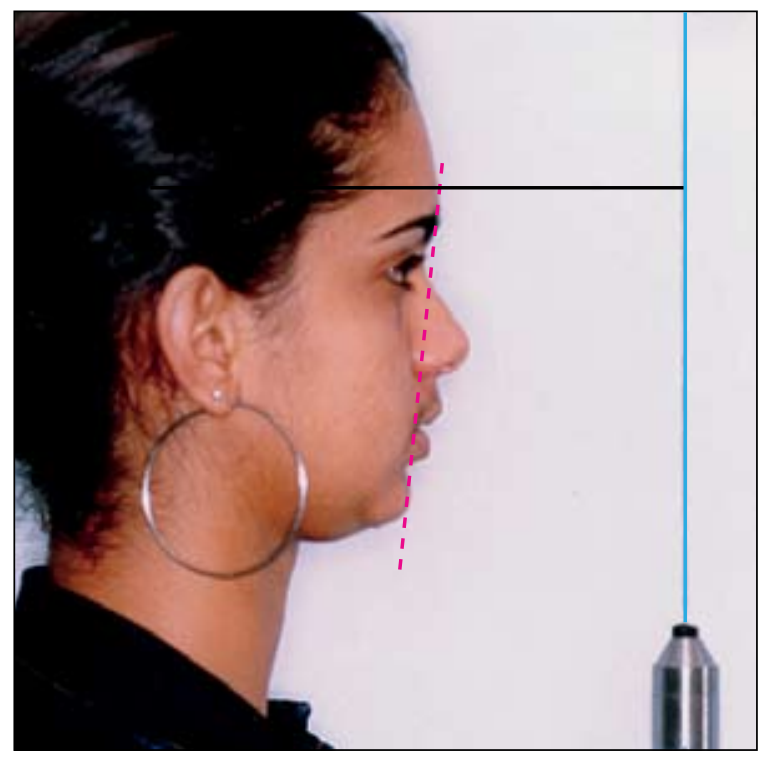

FIGURA 1 - Técnica para obtenção dos traçados nas fotografias laterais. 
Demarcamos os pontos superior e inferior da corrente metálica do prumo, representando a linha vertical verdadeira (VER).

Baseando-se nos métodos de registro da PNC com as fotografias nesta postura, realizamos uma modificação dos pontos do perfil mole do paciente, uasdos por Lundstrom et al. $26,27,28$, que utilizaram os pontos Násio Mole (N') e Pogônio Mole (Pog') para a linha do perfil mole. Porém, consideramos o ponto Násio mole suscetível à alteração pela compressão do posicionador Násio, na estabilização vertical da cabeça durante a tomada das telerradiografias laterais.

Uma linha de referência do perfil mole do paciente foi obtida passando pelos pontos Glabela (Gl), localizado na região frontal e não suscetível à alteração, e Pogônio Mole (Pog').

Uma perpendicular à linha vertical verdadeira (VER) foi traçada, passando pelo ponto Glabela, denominada de linha horizontal verdadeira (HOR).

As linhas do perfil mole (Gl-Pog'), horizontal verdadeira (HOR) e vertical verdadeira (VER) foram transferidas por transparência da fotografia para a telerradiografia em norma lateral.

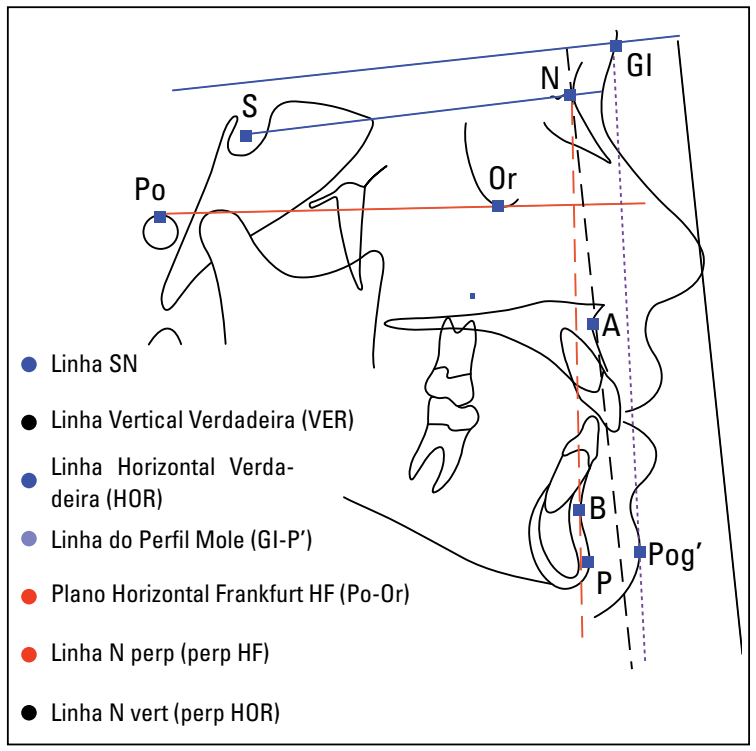

FIGURA 2 - Traçado do desenho anatômico, pontos cefalométricos, linhas e planos utilizados na telerradiografia lateral.

\section{Nas telerradiografias laterais}

Para a elaboração dos traçados e mensuração das grandezas cefalométricas foram utilizados: negatoscópio marca $\mathrm{VH}^{\circledR}$ Equipamentos; papel Ultraphan ${ }^{\circledR}(0,7 \mathrm{~mm}$ de espessura, medindo 17,5 x $17,5 \mathrm{~cm}$ ), fita adesiva transparente, lapiseira com grafite de diâmetro $0,5 \mathrm{~mm}$, borracha macia, régua milimetrada, esquadro, transferidor e papel cartão de cor preta para máscara de isolamento da luz.

- Traçado do desenho anatômico (Fig. 2)

Sobre cada telerradiografia foi adaptada uma folha de papel Ultraphan ${ }^{\circledR}$, fixada com fita adesiva transparente e depois colocada sobre um negatoscópio em ambiente escurecido, onde o traçado foi realizado. Em seguida, os pontos cefalométricos foram anotados para que as linhas e planos pudessem ser orientados.

- Obtenção e interpretação das grandezas cefalométricas (Fig. 3):

- HOR.HF: ângulo formado pelo plano horizontal de Frankfurt (HF) e a linha horizontal verdadeira (HOR).

- Nperp-P: distância linear entre a linha Nperp e o ponto $P$.

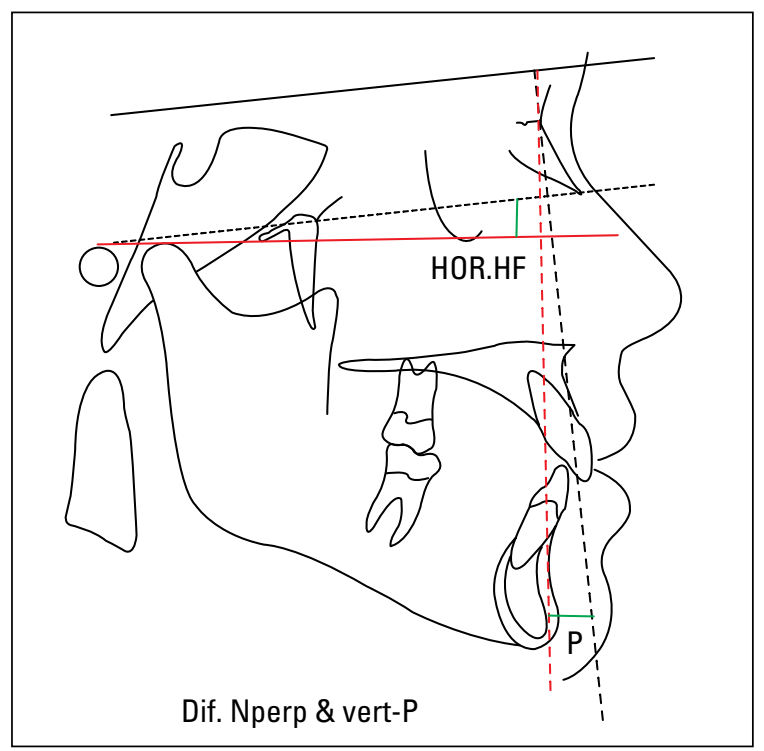

FIGURA 3 - Obtenção e interpretação das grandezas cefalométricas nas telerradiografias laterais. 
- Nvert-P: distância linear entre a linha Nvert e o ponto $P$.

- Dif. Nperp \& vert-P (Diferença entre NperpP e Nvert-P): comparação da diferença no diagnóstico da posição ântero-posterior da mandíbula, utilizando a posição natural da cabeça e sua linha de referência (Nvert), com a posição da cabeça utilizando o plano horizontal de Frankfurt paralelo ao solo e sua linha de referência (Nperp).

As radiografias foram analisadas sempre pelo mesmo observador, para avaliar as variações das grandezas cefalométricas citadas entre os dois grupos, ou seja, com padrão ortopédico facial de Classe I e Classe II, e os respectivos resultados foram submetidos aos testes estatísticos.

\section{Análise estatística}

Os dados obtidos foram apresentados em média e desvio-padrão, tendo sido submetidos aos testes de normalidade de Shapiro-Wilk e D'Agostino e Pearson. As variáveis medidas nos dois grupos foram comparadas em conjunto e isoladamente com o valor padrão "0" empregando-se o teste $t$ para amostras isoladas, tendo sido também calculado o coeficiente de variação das amostras. O teste $t$ de Student para valores não pareados foi empregado na comparação entre as médias observadas nos dois grupos. A relação entre os valores do ângulo HOR.HF e da diferença entre Nperp-P e Nvert-P foi avaliada através de regressão linear. Os valores de probabilidade menores do que 0,05 foram considerados significativos.

\section{RESULTADOS}

Os resultados das medidas empregadas na avaliação do trabalho estão representados nas tabelas 1 e 2 . A tabela 1 apresenta as medidas individuais dos 30 indivíduos do grupo I, o qual apresentou padrão esquelético facial de Classe I, e a tabela 2 apresenta as medidas dos 30 indivíduos do grupo II, o qual apresentou padrão esquelético facial de Classe II com retrusão mandibular.

As tabelas 1 e 2 relacionam o número do pa- ciente, a idade, os valores dos ângulos ANB e SNB para a classificação do padrão esquelético facial conforme a idade, o valor do ângulo HF.HOR para avaliação da inclinação da posição natural da cabeça através da linha horizontal verdadeira (HOR) em relação ao plano horizontal de Frankfurt (HF), e a diferença entre Nperp e Nvert ao ponto P, a qual significa a distância linear em milímetros da diferença no diagnóstico da posição sagital mandibular entre a posição natural da cabeça e o plano horizontal de Frankfurt.

O ângulo HF. HOR apresenta um valor positivo quando o plano de Frankfurt (HF) passa acima da linha horizontal verdadeira (HOR). Isto significa que a posição natural da cabeça apresenta uma inclinação maior no sentido anti-horário (extensão) do que o plano de Frankfurt paralelo ao solo. Esta extensão da cabeça proporciona uma posição mais avançada do mento da mandíbula, resultando na diferença de diagnóstico mandibular verificada pelo valor positivo da grandeza dif. Nperp \& vert-P. $\mathrm{O}$ valor negativo de HF.HOR relaciona uma inclinação da posição natural da cabeça no sentido horário (flexão) em relação ao plano horizontal de Frankfurt, resultando em uma posição mais retruída da mandíbula, verificada pelo valor da grandeza dif. Nperp \& vert-P, sendo este negativo.

A idade dos pacientes estudados variou de 8 a 37 anos, com média de 22,8 anos e desvio padrão de 6,9 anos.

Os pacientes do grupo I apresentaram valores de ANB que variaram de $0^{\circ}$ a $5^{\circ}$, com média de $2,16^{\circ}$ e desvio padrão de $1,56^{\circ}$, e valores de SNB que variaram de $74,5^{\circ}$ a $83,5^{\circ}$, com média de $78,3^{\circ}$ e desvio padrão de $1,97^{\circ}$. Os valores de ANB nos pacientes do grupo II variaram de $4,5^{\circ}$ a $10^{\circ}$, com média de $5,81^{\circ}$ e desvio padrão de $1,17^{\circ}$, e os valores de SNB variaram de $71^{\circ}$ a $76^{\circ}$, com média de $74,4^{\circ}$ e desvio padrão de $1,4^{\circ}$.

A variável ângulo HOR.HF apresentou um valor mínimo de $-11,5^{\circ}$ e máximo de $8^{\circ}$ em todos os 60 casos estudados. O valor médio observado em toda a amostra foi de $-0,175^{\circ}$, com um 
Tabela 1 - GRUPO 1: Padrão esquelético Classe I - idade, valores dos ângulos ANB, SNB, HF. HOR e da distância em mm da grandeza dif. Nperp \& vert-P.

\begin{tabular}{|c|c|c|c|c|c|}
\hline $\begin{array}{c}n^{0} \\
\text { indivíduo }\end{array}$ & Idade & ANB & SNB & HF.HOR & $\begin{array}{c}\text { dif. Nperp } \\
\& \text { vert-P }\end{array}$ \\
\hline 1 & $30 \mathrm{a} 0 \mathrm{~m}$ & 3,5 & 77 & $-11,5$ & $-25,5$ \\
\hline 2 & $16 a 1 m$ & 0,5 & 77 & -7 & -14 \\
\hline 3 & 13a3m & 4 & 77 & -1 & $-1,5$ \\
\hline 4 & $13 \mathrm{a} 0 \mathrm{~m}$ & 2,5 & 79 & $-1,5$ & -3 \\
\hline 5 & $17 \mathrm{a} 10 \mathrm{~m}$ & 0 & 79 & $-4,5$ & -9 \\
\hline 6 & $16 \mathrm{a} 1 \mathrm{~m}$ & 1 & 77 & 5,5 & 11,5 \\
\hline 7 & $28 \mathrm{a} 9 \mathrm{~m}$ & 0 & 78 & 2 & 4,5 \\
\hline 8 & $11 \mathrm{a} 2 \mathrm{~m}$ & 5 & 78 & $-0,5$ & -1 \\
\hline 9 & $14 a 4 m$ & 0 & 78 & -1 & -2 \\
\hline 10 & $19 a 2 m$ & 3,5 & 83,5 & 2 & 2,5 \\
\hline 11 & 10a1m & 5 & 75 & -1 & -2 \\
\hline 12 & $16 a 6 m$ & 3 & 82 & 1 & 2,5 \\
\hline 13 & 27a1m & 0 & 80 & $-4,5$ & -10 \\
\hline 14 & 31a1m & 0 & 82 & 3 & 6,5 \\
\hline 15 & 26a1m & 3 & 77 & $-1,5$ & -3 \\
\hline 16 & $21 \mathrm{a} 2 \mathrm{~m}$ & 0,5 & 80 & 4 & 9 \\
\hline 17 & 09a2m & 4 & 78 & $-1,5$ & $-1,4$ \\
\hline 18 & $37 a 6 m$ & 1 & 77 & 2,5 & 6 \\
\hline 19 & $31 \mathrm{a7m}$ & 2 & 77 & 7,5 & 15,5 \\
\hline 20 & 08a3m & 2 & 74,5 & 7,5 & 15 \\
\hline 21 & 27a2m & 3 & 77,5 & 2 & 3 \\
\hline 22 & $22 \mathrm{a} 0 \mathrm{~m}$ & 2 & 78,5 & $-1,5$ & $-3,5$ \\
\hline 23 & $19 \mathrm{a} 6 \mathrm{~m}$ & 2,5 & 79,5 & -4 & -9 \\
\hline 24 & $28 \mathrm{a} 5 \mathrm{~m}$ & 3,5 & 80 & 2 & 4 \\
\hline 25 & $22 \mathrm{a} 0 \mathrm{~m}$ & 1,5 & 77 & -3 & -7 \\
\hline 26 & $30 \mathrm{a} 2 \mathrm{~m}$ & 1,5 & 78 & 4,5 & 9,5 \\
\hline 27 & $27 a 2 m$ & 2,5 & 78 & -4 & -9 \\
\hline 28 & $25 a 3 m$ & 0,5 & 79 & -3 & $-6,5$ \\
\hline 29 & $18 \mathrm{a} 5 \mathrm{~m}$ & 3,5 & 79,5 & 2 & 3 \\
\hline 30 & 13a3m & 4 & 76 & -1 & -2 \\
\hline
\end{tabular}

desvio padrão de $3,741^{\circ}$. Os dados observados não apresentam diferença estatisticamente significante em relação ao valor padrão "0" e o coeficiente de variação da amostra foi de $2.137 \%$.

Os resultados observados para a variável ângulo HOR.HF isoladamente nos dois grupos estudados são apresentadas na tabela 3 .
Tabela 2 - GRUPO 2: Padrão esquelético Classe II - idade, valores dos ângulos ANB, SNB, HF. HOR e da distância em $\mathrm{mm}$ da grandeza dif. Nperp \& vert-P.

\begin{tabular}{|c|c|c|c|c|c|}
\hline $\begin{array}{c}\mathrm{n}^{\mathbf{0}} \\
\text { indivíduo }\end{array}$ & Idade & ANB & SNB & HF.HOR & $\begin{array}{l}\text { dif. Nperp } \\
\text { \& vert-P }\end{array}$ \\
\hline 1 & $28 \mathrm{a} 4 \mathrm{~m}$ & 6 & 74 & 1,5 & 2,5 \\
\hline 2 & $22 \mathrm{a} 10 \mathrm{~m}$ & 4,5 & 75 & 2 & 4,5 \\
\hline 3 & $24 a 4 m$ & 7 & 71 & -1 & $-1,5$ \\
\hline 4 & $25 \mathrm{a} 9 \mathrm{~m}$ & 6 & 76 & $-0,5$ & -1 \\
\hline 5 & $30 a$ & 5,5 & 73,5 & 8 & 21 \\
\hline 6 & $28 \mathrm{a} 7 \mathrm{~m}$ & 4,5 & 75,5 & -1 & -2 \\
\hline 7 & $20 \mathrm{a} 2 \mathrm{~m}$ & 5,5 & 72 & 0 & 0 \\
\hline 8 & $16 \mathrm{a} 1 \mathrm{~m}$ & 5 & 76 & 1,5 & 2,5 \\
\hline 9 & $19 \mathrm{a} 4 \mathrm{~m}$ & 6 & 75,5 & -7 & $-14,5$ \\
\hline 10 & 9a8m & 10 & 73 & 6 & 11 \\
\hline 11 & 22a9m & 5,5 & 74 & $-4,5$ & -9 \\
\hline 12 & $31 \mathrm{a} 3 \mathrm{~m}$ & 8,5 & 72 & 2 & 4,5 \\
\hline 13 & $29 \mathrm{a} 10 \mathrm{~m}$ & 5 & 74,5 & 1 & 1,5 \\
\hline 14 & $26 a$ & 6 & 75 & 0 & 0 \\
\hline 15 & $24 \mathrm{a} 11 \mathrm{~m}$ & 6,5 & 75,5 & 0,5 & 1 \\
\hline 16 & $28 \mathrm{a} 1 \mathrm{~m}$ & 5 & 74,5 & 1 & 1,5 \\
\hline 17 & $25 \mathrm{a} 10 \mathrm{~m}$ & 5,5 & 76 & 2 & 4 \\
\hline 18 & $19 \mathrm{a} 7 \mathrm{~m}$ & 4,5 & 74,5 & 2 & 4,5 \\
\hline 19 & $24 \mathrm{a} 3 \mathrm{~m}$ & 6 & 75,5 & -1 & -2 \\
\hline 20 & $32 \mathrm{a} 9 \mathrm{~m}$ & 7 & 75 & -1 & $-2,5$ \\
\hline 21 & $33 a$ & 5 & 75,5 & -4 & -8 \\
\hline 22 & $28 \mathrm{a} 2 \mathrm{~m}$ & 5,5 & 76 & $-4,5$ & -9 \\
\hline 23 & $24 \mathrm{a} 1 \mathrm{~m}$ & 5 & 73 & 0 & 0 \\
\hline 24 & $23 \mathrm{a} 7 \mathrm{~m}$ & 6 & 74 & -3 & -7 \\
\hline 25 & $33 \mathrm{a} 7 \mathrm{~m}$ & 6 & 74,5 & -4 & -9 \\
\hline 26 & $19 \mathrm{a} 6 \mathrm{~m}$ & 5,5 & 72 & 5 & 10 \\
\hline 27 & $21 \mathrm{a} 2 \mathrm{~m}$ & 4,5 & 74 & 2 & 5 \\
\hline 28 & $21 \mathrm{a} 4 \mathrm{~m}$ & 6,5 & 76 & -2 & -4 \\
\hline 29 & $30 \mathrm{a} 3 \mathrm{~m}$ & 6 & 76 & -6 & -11 \\
\hline 30 & $31 \mathrm{a} 4 \mathrm{~m}$ & 5 & 75 & 1 & 1,5 \\
\hline
\end{tabular}

Os dados observados nos dois grupos também não apresentam diferenças estatisticamente significantes em relação ao valor padrão "0".

O gráfico 1 mostra a comparação dos valores do ângulo HOR.HF nos dois grupos estudados, bem como a dispersão dos valores, não tendo sido observada diferença significativa entre eles através 
Tabela 3 - Média, desvio-padrão, valor máximo e mínimo e coeficiente de variação do ângulo HOR.HF nos grupos Classe I e II.

\begin{tabular}{ccc}
\hline & Classe I & Classe II \\
\cline { 2 - 3 } Média & $-0,216$ & $-0,133$ \\
Desvio-padrão & 4,147 & 3,358 \\
Valor mínimo & $-11,5$ & -7 \\
Valor máximo & 7,5 & 8 \\
Coeficiente de & $1913 \%$ & $2518 \%$ \\
Variação & & \\
\hline
\end{tabular}

do teste $t$ de Student.

A variável Diferença entre Nperp-P e Nvert-P apresentou um valor mínimo de $-25,5 \mathrm{~mm}$ e máximo de $21 \mathrm{~mm}$ em todos os 60 casos estudados. O valor médio observado em toda a amostra foi de $-0,416 \mathrm{~mm}$, com um desvio padrão de $7,972 \mathrm{~mm}$. Os dados observados não apresentam diferença estatisticamente significante em relação ao valor padrão "0" e o coeficiente de variação da amostra foi de $1913 \%$.

Os resultados observados para a variável Diferença entre Nperp-P e Nvert-P isoladamente nos dois grupos estudados são apresentadas na tabela 4.

Os dados observados nos dois grupos não apresentam diferenças estatisticamente significantes em relação ao valor padrão "0".

O gráfico 2 mostra a comparação dos valores

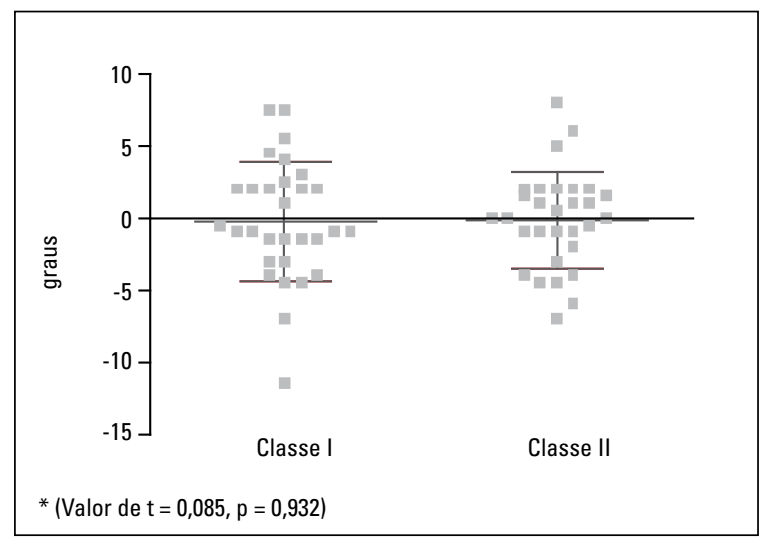

GRÁFICO 1 - Média, desvio-padrão e dispersão do ângulo HOR.HF nos grupos Classe I e II.
Tabela 4 - Média, desvio-padrão, valor máximo e mínimo e coeficiente de variação da grandeza Dif. Nperp \& vert-P nos grupos I e II.

\begin{tabular}{ccc}
\hline & Classe I & Classe II \\
\cline { 2 - 3 } Média & $-0,65$ & $-0,183$ \\
Desvio-padrão & 8,795 & 7,201 \\
Valor mínimo & $-25,5$ & $-14,5$ \\
Valor máximo & 15,5 & 21 \\
Coeficiente de & $1356 \%$ & $3927 \%$ \\
Variação & & \\
\hline
\end{tabular}

da Diferença entre Nperp-P e Nvert-P nos dois grupos estudados, bem como a dispersão dos valores, não tendo sido observada diferença significativa entre eles através do teste $t$ de Student.

A relação observada entre as variáveis ângulo HOR.HF e Diferença entre Nperp-P e Nvert-P apresenta um comportamento linear, com um coeficiente de determinação $\left(\mathrm{R}^{2}\right)$ de 0,989 e um valor de $\mathrm{p}<0,0001$.

A equação que define a relação observada pode ser expressa através do coeficiente de 2,119 \pm 0,029 para o cálculo estimado da Diferença entre Nperp-P e Nvert-P a partir do ângulo HOR.HF (Gráf. 3).

\section{DISCUSSÃO}

A Ortodontia tem enfatizado que a análise clínica do perfil facial é a chave do diagnóstico,

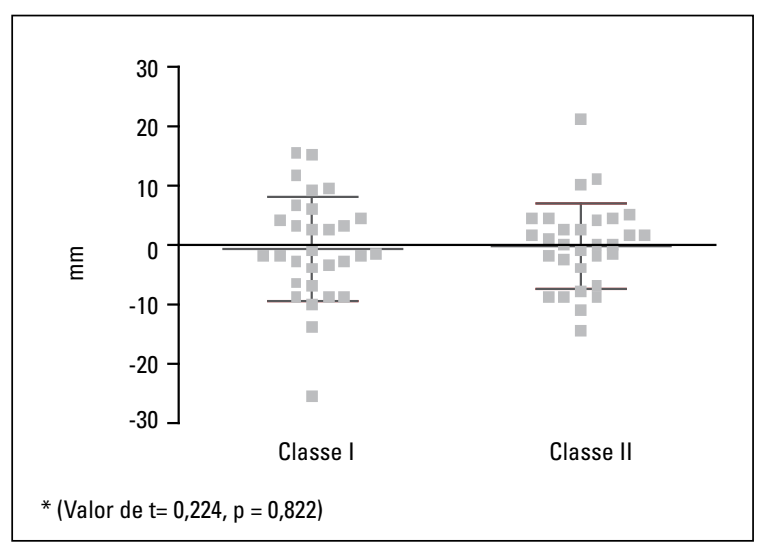

GRÁFICO 2 - Média, desvio-padrão e dispersão da Dif. Nperp \& vert-P nos grupos Classe I e II. 


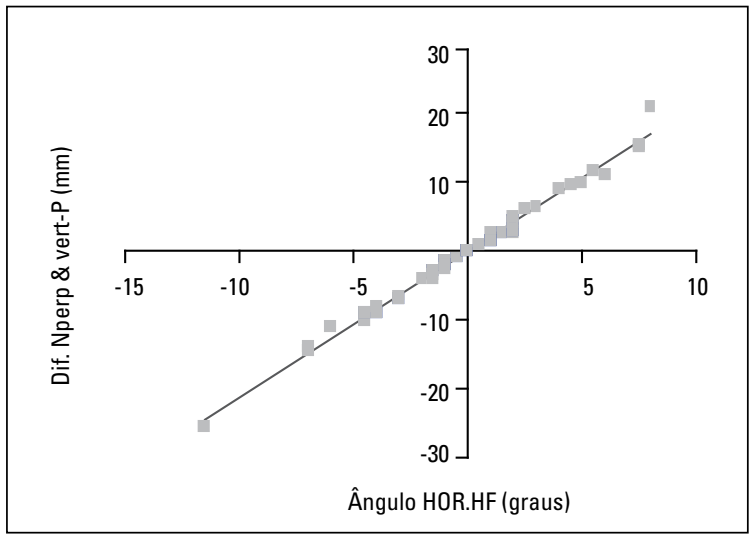

GRÁFICO 3 - Correlação entre Dif. Nperp \& vert-P e HOR.HF nos grupos Classe I ell.

em contrapartida da inerente desvantagem da utilização das análises cefalométricas baseadas em linhas de referência intracranianas com grande variabilidade, comprometendo o diagnóstico e o plano de tratamento ortodôntico.

Quando Schmidt (1876 apud MOORREES, $\mathrm{KEAN}^{38}$, 1958) observou que, para se encontrar o posicionamento horizontal da cabeça, seria necessário observar as pessoas, determinando, primeiramente, qual o plano horizontal transverso que a cabeça assume em posição normal, o plano horizontal de Frankfurt (Po-Or) estava sendo provavelmente a mais conhecida e aceita aproximação da linha horizontal fisiológica.

Desde 1931, com o desenvolvimento da cefalometria radiográfica e o advento da multiplicação das análises cefalométricas, o plano horizontal de Frankfurt, que era utilizado como parâmetro para as mensurações craniométricas, passou a servir tanto para orientar a posição da cabeça no cefalostato, quanto como referência para avaliação do comprometimento dento-esquelético em várias análises cefalométricas, como as de Tweed, Downs e McNamara Jr.

O conceito de posição natural da cabeça (PNC) foi introduzido na Ortodontia na década de $50^{4,14,15,36}$. Downs ${ }^{14,15}$ relacionou o plano horizontal verdadeiro com o plano horizontal de Frankfurt e encontrou uma pequena diferen- ça média de $1,3^{\circ}$ (desvio padrão de $5^{\circ}$ ), porém, quando havia discrepância entre as medidas cefalométricas e suas avaliações clínicas, observou que o plano horizontal de Frankfurt desviava-se da horizontal verdadeira. $\mathrm{O}$ autor questionou se um desvio do plano horizontal de Frankfurt em $10^{\circ}$ para cima ou para baixo conduziria a interpretações incorretas do padrão facial. Relatou a imprecisão na localização do ponto Pório e Orbitário, induzindo a discrepâncias na utilização do plano horizontal de Frankfurt, verificada também por outros autores ${ }^{36,56}$.

Krogman ${ }^{22}$ obteve diagnósticos cefalométricos utilizando 45 análises cefalométricas diferentes e verificou que, no que tange a posição ântero-posterior da maxila e da mandíbula em relação à base do crânio, as análises que utilizaram como referência o plano horizontal de Frankfurt diagnosticaram uma protrusão maxilar associada a uma posição correta da mandíbula, ao passo que as que tinham como referência a linha Sela - Násio consideraram haver uma boa posição da maxila e uma retrusão da mandíbula. Dependendo da escolha da linha de referência cefalométrica, poderia apresentar resultados marcadamente diferentes.

A variação do ângulo formado entre o plano horizontal de Frankfurt e a linha Sela-Násio (SN.PoOr) foi medida em vários estudos. A diferença entre estas linhas de referência deveria não apresentar variação significante para ser considerada estável e confiável.

Moore $^{35}$ encontrou uma variação tão alta do ângulo SN.PoOr em casos de oclusão normal e perfil equilibrado, a ponto de justificar a utilização de valores normativos distintos de SNA e SNB, segundo a variação do ângulo SN. PoOr.

Proffit ${ }^{43}$ sugeriu que, previamente à utilização de medidas angulares compostas pela linha Sela-Násio para o diagnóstico cefalométrico, deve-se avaliar o ângulo entre a linha SN e o plano horizontal de Frankfurt, ajustando os valores de SNA e SNB.

Desta maneira, a utilização de uma referência extra-craniana associada à posição natural da 
cabeça para estabelecer um posicionamento estável e reprodutível nas avaliações da estética facial real no diagnóstico e planejamento ortodôntico, independentemente das angulações dos planos de referência intracranianos, padronizaria o posicionamento da cabeça para as análises cefalométricas e clínicas.

Bjerin ${ }^{4}$ utilizou a visualização das pupilas num espelho e de uma corrente metálica unida a um prumo posicionada à margem anterior do chassi porta-filme, possível de ser visualizada à frente do contorno do perfil facial na telerradiografia lateral. Esta linha extra-craniana foi denominada vertical verdadeira (VER). A partir desta referência vertical, podia-se traçar uma perpendicular, obtendo-se a linha denominada horizontal verdadeira (HOR). Em seu estudo radiográfico e fotográfico utilizando sobreposições, avaliou a reprodutibilidade da PNC como referência extracraniana. Os valores referentes ao desvio-padrão foram de $1,62^{\circ}$ para o registro na posição sentada e de $1,34^{\circ}$ para a posição em pé. A variação da inclinação do plano horizontal de Frankfurt em relação à linha horizontal verdadeira (HOR) foi de $4,6^{\circ}$ em pé e $4,3^{\circ}$ sentado.

As olivas do cefalostato introduzidas nos meatos acústicos alterariam a posição da cabeça ao se registrar a postura natural, pois poderiam impor limitação física no posicionamento e alterar as dimensões angulares da cabeça e do pescoço ${ }^{12,20,57,67,68}$.

As comparações dos métodos com e sem a utilização do espelho na obtenção da PNC relataram menor variação com o uso do mesmo ${ }^{12,60}$.

Segundo Usumez e Orhan ${ }^{63}$, os métodos de obtenção da PNC como rotina em telerradiografias laterais resultaria num maior consumo de tempo e necessidade de treinamento específico do técnico, do clínico e do paciente, em relação ao método convencional. Poucos clínicos realizam a obtenção de telerradiografias em PNC devido à falta de um método que possa ser utilizado facilmente e com precisão pelos radiologistas ${ }^{21}$. Porém, a obtenção da PNC para registros fotográficos é um método mais simples e prático, facilmente transferível para as telerradiografias em norma lateral e utilizado por vários autores.

Ressaltamos que o método fotográfico para registro da PNC foi utilizado neste estudo por considerarmos o mesmo mais preciso, pois ao contrário do que possa ocorrer durante o posicionamento do paciente no cefalostato, durante a tomada radiográfica, teremos uma posição mais relaxada e com menores influências na obtenção da PNC. Outra vantagem é a fidelidade na análise do perfil facial para diagnóstico e planejamento, utilizandose a fotografia na posição da cabeça com inclinação na qual o paciente se conduz no seu dia a dia, expressando a real estética facial na avaliação das alterações e compensações faciais.

F. Lundstrom e A. Lundstrom ${ }^{26,28}$ utilizaram o método fotográfico para registrar a PNC, realizando duas fotografias seguidas dos pacientes em pé e relaxados, olhando para suas pupilas no espelho vertical localizado a 1 metro de distância à sua frente. Uma linha de chumbo foi utilizada para definir a linha vertical verdadeira (VER) nas fotografias. Uma linha horizontal (HOR), perpendicular à vertical foi traçada, assim como uma linha do perfil mole unindo os pontos Násio e Pogônio (N'- Pog') do paciente. As linhas HOR e VER foram transferidas da fotografia para a telerradiografia, através dos ângulos entre a linha N'-Pog' e as linhas HOR e VER. Uma diferença de $1^{\circ}$ foi encontrada entre a primeira e a segunda fotografia na PNC, indicando a reprodutibilidade deste método.

A importância da reprodutibilidade da PNC foi observada por muitos pesquisadores, possibilitando a utilização de linhas extra-cranianas na avaliação cefalométrica e para se verificar a confiabilidade das linhas de referência intracranianas. 4,9,12,18,23,26,29,38,41,42,45,49,50,53,60,65,68.

Leitão e $\mathrm{Nanda}^{23}$ estudaram uma amostra de 284 adultos do gênero masculino (Os registros fotográficos e cefalométricos foram obtidos com a PNC) e avaliaram que a variação média do plano 
horizontal de Frankfurt e do plano palatino em relação à linha HOR era menor que $1,0^{\circ}$.

Estudos longitudinais, realizados por Cooke ${ }^{11}$; Peng e Cooke ${ }^{41}$, confirmaram a reprodutibilidade da PNC, abrangendo períodos de 5 e 15 anos, respectivamente.

Uma referência vertical através do ponto Násio foi inicialmente apresentada por McNamara $\mathrm{Jr}^{33}$, utilizando uma perpendicular a Frankfurt (Nperp). Viazis $^{67}$ considerou a linha horizontal verdadeira (HOR) mais confiável que o plano horizontal de Frankfurt na avaliação da posição ântero-posterior das bases apicais, relacionando a perpendicular à HOR passando pelo ponto Násio (Nvert), mais precisa para o planejamento, principalmente em tratamentos cirúrgicos.

Proffit e White ${ }^{44}$ propuseram a utilização de uma perpendicular do Násio com a horizontal verdadeira (HOR) na avaliação da relação ânteroposterior da maxila e da mandíbula, com medidas lineares. Três medidas lineares foram sugeridas dos pontos $\mathrm{A}, \mathrm{B}$ e Pog à perpendicular da $\mathrm{HOR}$, passando pelo ponto $\mathrm{N}$, denominada NperpHOR (Nvert).

A alteração sagital da rotação da cabeça para cima ou para baixo, alterando sua inclinação em relação à vertical verdadeira é muito mais expressiva na região anterior da mandíbula do que na maxila, em conseqüência da posição do centro de rotação da cabeça.

Leitão e $\mathrm{Nanda}^{23}$, avaliando a posição sagital da mandíbula através da vertical verdadeira por Nvert-B e Nvert-P, encontraram uma alta variação dos valores destas grandezas.

Aqueles que apresentaram uma variação individual extrema da inclinação da cabeça, em relação à média, foram divididos em grupos de flexores e extensores da cabeça. Segundo Marcotte ${ }^{30}$; Showfety et al. ${ }^{56}$; Solow et al. ${ }^{58}$; Solow e Tallgren ${ }^{61}$; Leitão e Nanda ${ }^{23}$, os extensores (inclinação posterior) apresentaram uma tendência a faces mais longas e retrognatas, com mandíbulas menores, indicando a possibilidade da PNC minimizar a discrepância mandibular.

Bjork ${ }^{6}$ citou uma tendência da postura da cabeça camuflar a sua morfologia. Baseado nesta afirmação, seria esperada uma tendência maior de inclinação posterior da cabeça em pacientes com padrão facial de Classe II apresentando retrusão mandibular.

Vig et al ${ }^{69}$ realizaram estudos sobre a respiração bucal e os resultados indicaram que, quando há obstrução nasal total pelo uso de um tampão, sempre ocorre a extensão na posição da cabeça. Linder-Aronson ${ }^{25}$ demonstrou a desinclinação da cabeça após a remoção da obstrução respiratória.

Cooke e Wei ${ }^{13}$ aplicaram as análises convencionais em chineses do gênero masculino e revelaram um padrão esqueletal de Classe II, com retrognatismo mandibular, quando comparados com caucasianos do mesmo gênero. No entanto, quando observados em posição natural da cabeça e utilizando a linha horizontal verdadeira como referência, o padrão esqueletal dos chineses passou a ser de Classe III. Os autores observaram que métodos convencionais poderiam resultar em erros significativos no diagnóstico e na elaboração do plano de tratamento.

No afã de estudarmos a variação da linha horizontal verdadeira (HOR), obtida em PNC, com o plano horizontal de Frankfurt (HF), utilizamos a grandeza angular HOR.HF.

Selecionamos uma amostra composta de 2 grupos de 30 indivíduos, divididos conforme o padrão do esqueleto facial em Classe I (harmonioso) e Classe II com retrusão mandibular, para verificarmos as inclinações da cabeça em ambos. Utilizamos os dados cefalométricos, verificados pelas grandezas ANB e SNB, na avaliação dos padrões faciais conforme a idade, segundo Martins ${ }^{31}$, para leucodermas brasileiros nesta seleção. Foram excluídos da amostra os indivíduos com alterações respiratórias para não interferir no posicionamento da cabeça devido a alterações funcionais ${ }^{25,69}$.

A variável ângulo HOR.HF apresentou um valor mínimo de $-11,5^{\circ}$ e máximo de $8^{\circ}$ em todos 
os 60 casos estudados. $\mathrm{O}$ valor médio observado em toda a amostra foi de $-0,175^{\circ}$, com um desvio padrão de $3,741^{\circ}$. Os dados observados não apresentam diferença estatisticamente significante em relação ao valor padrão " 0 " e o coeficiente de variação da amostra foi de $2.137 \%$. Na comparação dos valores do ângulo HOR. HF nos dois grupos estudados não foi observada diferença significativa entre eles através do teste $t$ de Student.

Contrariamente aos resultados relatados por Bjork $^{6}$; Marcotte ${ }^{30}$; Showfety et al. ${ }^{56}$; Solow et al. ${ }^{58}$; Solow, Tallgren ${ }^{61}$; Leitão e Nanda ${ }^{23}$, não houve diferença entre as variáveis que determinam a posição natural da cabeça entre os padrões faciais de Classe I e Classe II com retrusão mandibular, em nossa amostra.

Apesar da diferença média da linha horizontal verdadeira (HOR), obtida em PNC, com o plano horizontal de Frankfurt apresentar-se estatisticamente não significante em ambos os grupos, houve uma acentuada variação individual, tanto no sentido horário como anti-horário (flexão e extensão), da inclinação da cabeça. Considerando os resultados desta amostra, a PNC independe do padrão esquelético facial e deveria ser prioritária sua avaliação individual, principalmente nos casos apresentando discrepância entre a linha horizontal verdadeira (HOR) e o plano horizontal de Frankfurt (HF).

A grandeza Diferença entre Nperp-P e Nvert$\mathrm{P}$ avalia a discrepância no diagnóstico da posição sagital da mandíbula, quando utilizamos a linha horizontal verdadeira (HOR) em PNC e o plano horizontal de Frankfurt (HF), respectivamente. O contorno anterior da sínfise da mandíbula, verificado pelo ponto Pogônio ( $\mathrm{P}$ ou Pog), sofre alteração em sua posição sagital em relação à vertical verdadeira, muito expressiva na rotação da cabeça para cima ou para baixo. Ao verificarmos a variável Diferença entre Nperp-P e Nvert-P, a mesma apresentou um valor mínimo de $-25,5 \mathrm{~mm}$ e máximo de $21 \mathrm{~mm}$ em todos os 60 casos estudados. O valor médio observado em toda a amostra foi de
$-0,416 \mathrm{~mm}$, com um desvio padrão de $7,972 \mathrm{~mm}$. Os dados observados não apresentaram diferença estatisticamente significante em relação ao valor padrão "0" e o coeficiente de variação da amostra foi de $1913 \%$. A variação da PNC, verificada pelo ângulo HF.HOR provoca influência equivalente sobre a posição sagital da mandíbula, comparando-se a diferença linear das grandezas Nperp-P e Nvert-P, não apresentando diferença estatisticamente significante entre os grupos de padrão facial de Classe I e Classe II.

Nos casos onde verificamos variação positiva do ângulo HF.HOR, houve inclinação posterior da cabeça (extensão), e a posição sagital mandibular apresentou-se mais protruída em PNC, verificada pela Dif. Nperp \& vert-P com valor positivo. Quando houve variação negativa do ângulo HF. HOR com inclinação anterior da cabeça (flexão), a posição sagital mandibular apresentou-se mais retruída em PNC, resultando em um valor negativo da grandeza Dif. Nperp \& vert-P.

A relação observada da variação do ângulo HOR.HF para o cálculo estimado da Diferença entre Nperp-P e Nvert-P foi através do coeficiente de $1: 2,119 \pm 0,029$. Esta discrepância do diagnóstico sagital mandibular compromete o planejamento ortodôntico nos pacientes que apresentaram diferença entre os planos horizontais intra e extra-cranianos. A utilização de linhas de referência cefalométricas intracranianas nem sempre reflete o padrão facial correto e, além disso, mostrou grande amplitude de variação nos indivíduos com configurações faciais similares.

Em busca da excelência no diagnóstico das anomalias maxilo-mandibulares aplicadas aos tratamentos ortodôntico-ortopédico-cirúrgicos, destacamos a importância das avaliações faciais e cefalométricas em norma lateral serem realizadas em posição natural da cabeça, utilizando as linhas horizontal e vertical verdadeiras como referências. Portanto, seria sensato utilizarmos a PNC como rotina, pois esta é a aparência com que somos vistos pelos outros em nosso dia a dia. 


\section{CONCLUSÕES}

Baseado na metodologia empregada e nos resultados estatísticos obtidos neste estudo, concluímos que:

- A posição natural da cabeça nos padrões faciais de Classe I e Classe II, verificada pela diferença entre a linha horizontal verdadeira e o plano horizontal de Frankfurt, apresenta acentuada variação individual, embora esta diferença em média não seja estatisticamente significante. Não houve diferença entre as variáveis que determinam a posição natural da cabeça entre os padrões faciais de Classe I e Classe II.
- A posição natural da cabeça resultou numa influência equivalente sobre a posição sagital da mandíbula, comparando-se a diferença linear das grandezas Nperp-P e Nvert-P, pelo coeficiente de $1: 2,119 \pm 0,029$, não apresentando diferença estatisticamente significante entre os grupos de padrão facial de Classe I e Classe II.

\title{
Study of the natural head position in relation to the Frankfurt horizontal plane in mandibular evaluation of the Class I and Class II skeletal pattern
}

\begin{abstract}
Aim: The present study aimed to compare the influence of natural head position (NHP) on Frankfurt plane behavior, as well as mandibular position evaluation, in a sample comprised by Class I and Class II facial patterns subjects. Methods: profile photographs of NHP were taken with the patients in a relaxed standing position looking into the reflected image of their own eyes in a mirror placed $1 \mathrm{~m}$ in front of them. A plumb line was used to define the vertical plane (VER) on the photographs, and a line connecting soft tissue glabela and pogonion were transfered from the photograph to the lateral teleradiograph. A horizontal line (HOR) at a right angle to the vertical plane with the intracranial reference Frankfurt horizontal plane (HF) was used to assess the variability in relation between $\mathrm{HF}$ and NHP. The effects of the alterations of the NHP on the cephalometric measurements to evaluate the sagittal position of anterior contour of the mandibule (difference between Nperp-P and Nvert-P). The investigation was based on pre-treatment photographic and radiographic recordings of 60 patients selected in two groups based in the Class I and Class II skeletal pattern. Results and Conclusion: the results demonstrated a high and similar inter-individual variability between Frankfurt horizontal plane (HF) and a true horizontal line (HOR) in both groups. But the average reported a small mean difference among these references, no statistically significant and similar in both groups. The relation observed of the HOR.HF angle variation to the estimated calculation of the Difference between Nperp$P$ and Nvert-P was through the coefficient 1:2,119 $\pm 0,029$. This discrepancy of the sagittal mandibular diagnosis compromise orthodontic planning in the patients that showed difference between intra and extra-cranial horizontal planes. We emphasize the importance of the lateral facial and cephalometric evaluation to be accomplish in natural head position using the true horizontal and vertical planes as references.
\end{abstract}

Key words: Natural head position. Cephalometry. Diagnosis.

\section{REFERÊNCIAS}

1. ARNETT, W. G.; BERGMAN, R. T. Facial keys to orthodontic diagnosis and treatment planning. Part I. Am J Orthod Dentofacial Orthop, St. Louis, v. 103, no. 4, p. 299 - 312, 1993.

2. BEAN, L. R. et al. A simplified method of taking radiographs for cephalometric analysis. J Oral Surg, Chicago, v. 28, no. 9, p. $675-678,1970$.

3. BENCH, R. W. Growth of the cervical vertebrae as related to tongue, face, and denture behavior. Am J Orthod, St. Louis, v. 49 , no. 3, p. $183-214,1963$.

4. BJERIN, R. A comparison between the Frankfort horizontal and the sella turcica-nasion as reference plane in cephalometric analysis. Acta Odontol Scand, Stockholm, v. 15, no. 1, p. 1 -12, 1957. 
5. BJORK, A. Some biological aspects of prognathism and occlusion of teeth. Acta Odontol Scand, Stockholm, v. 9, no. 2, p. 1 $-40,1950$.

6. BJORK, A. Cranial base development. Am J Orthod, St. Louis, V. 41, no. 3, p. $198-225,1955$

7. BRODIE, A. G. Emerging concepts of facial growth. Angle Orthod, Appleton, v. 41, no. 2, p. 103-118, Apr. 1971

8. CARLSOO, S.; LEIJON, G. A radiographic study of the position of the hyo-laryngeal complex in relation to the skull and the cervical column in man. Trans $\mathbf{R}$ Sch Dent Stockh Umea, Umea, v. 5 , no. 2 , p. $13-34,1960$.

9. CLEALL, J. F.; ALEXANDER, W. J.; McINTYRE, H. M. Head posture and its relationship to deglutition. Angle Orthod, Appleton, v. 36, no. 4, p. 335-350, Oct. 1966.

10. COLE, S. C et al. Natural head position, posture, and prognathism: the Chapman prize essay, 1986. Br J Orthod, Oxford, v. 15, no. 4, p. 227-239, Nov. 1988.

11. COOKE, M. S. Five year reproducibility of natural head posture: a longitudinal study. Am J Orthod Dentofacial Orthop, St. Louis, v. 97, no. 6, p. 489-494, June 1990.

12. COOKE, M. S.; WEI, S. W. The reproducibility of natural head posture: a methodological study. Am J Orthod Dentofacial Orthop, St. Louis, v. 93, no. 4, p. 280-288, 1988.

13. COOKE, M. S.; WEI, S. W. A summary five - factor cephalometric analysis based on natural head posture and the true horizontal. Am J Orthod Dentofacial Orthop, St. Louis, v. 93, no. 3, p. 213-223, Mar. 1988

14. DOWNS, W. B. The role of cephalometrics in orthodontic case analysis and diagnosis. Am J Orthod, St. Louis, v. 38, no. 3, p. $162-182,1952$.

15. DOWNS, W. B. Analysis of dentofacial profile. Angle Orthod, Appleton, v. 26, no. 4, p. 191 - 212, 1956

16. FEUER, D. D. The value of the PM vertical reference line to estimate natural head position. Am J Orthod, St. Louis, v. 64, no. 2, p. 209-210, Aug. 1973.

17. FJELLVANG, H.; SOLOW, B. Craniocervical postural relations and craniofacial morphology in 30 blind subjects. Am J Orthod Dentofacial Orthop, St. Louis, v. 90, no. 4, p. 327-334, Oct. 1986.

18. FOSTER, T. D.; HOWAT, A. P.; NAISH, P. J. Variation in cephalometric reference lines. Br J Orthod, Oxford, v. 80, no. 4 p. $183-187$, Oct. 1981.

19. FRANKEL, R. The applicability of the occipital reference base in cephalometrics. Am J Orthod, St. Louis, v. 77, no. 4, p. 379-395, Apr. 1980

20. GREENFIELD, B.; KRAUS, S.; LAWRENCE, E.; WOLF, S. L. The influence of cephalostatic ear rods on the positions of the head and neck during postural recordings. Am J Orthod Dentofacial Orthop, St. Louis, v. 95, no. 4, p. 312- 318, 1989.

21. HUGGARE, J. A. V. A natural head position technique for radiographic cephalometry. Dentomaxillofac Radiol, Houndsmills, v. 22 , no. 2, p. 74-76, May 1993

22. KROGMAN, W. M. Craniometry and cephalometry as research tools in growth of head and face. Am J Orthod, St. Louis, v. 37 no. 6, p. 406-414, June 1951

23. LEITÃO, P.; NANDA, R. S. Relationship of natural head position to craniofacial morphology. Am J Orthod Dentofacial Orthop, St. Louis, v. 117, no. 4, p. 406- 417, Apr. 2000.

24. LINDER-ARONSON, S.; BACKSTROM, A. A comparison between mouth and nose breathers with respect to occlusion and facial dimensions. Odontol Rev, Lund, v. 11, no. 4, p. 343, 1960.

25. LINDER - ARONSON, S. The physiologic basis of functional appliances: The role of respiration. In: GRABER,T. M.; RAKOSI, T.; PETROVIC, A. G. Dentofacial Orthopedics with Funtional Appliances. 2nd ed. St. Louis: C. V. Mosby, 1997. cap. 3, p. 5 - 12.

26. LUNDSTROM, F.: LUNDSTROM, A. Natural head position as a basis for cephalometric analysis. Am J Orthod Dentofacial Orthop, St. Louis, v. 101, no. 3, p. 244-247, 1992.

27. LUNDSTROM, F. et al. Natural head position: basic considerations in cephalometric analysis. Eur J Orthod, Oxford, v. 17, no. 2, p. $111-120,1995$
28. LUNDSTROM, F.; LUNDSTROM, A. Clinical evaluation of maxillary and mandibular prognathism. Eur J Orthod, Oxford, v. 11 , no. 4 , p. $408-413,1989$

29. LUYK, N. H. et al. The reproducibility of the natural head position in lateral cephalometric radiographs. Br J Oral Maxillofac Surg, London, v. 24, no. 5, p. 357-366, Oct. 1986.

30. MARCOTTE, M. R. Head posture and dentofacial proportions. Angle Orthod, Appleton, v. 51, no. 3, p. 208 -214, July 1981.

31. MARTINS, D. R. Estudo comparativo dos valores cefalométricos das análises de Downs, Tweed, Steiner e Alabama, com os de adolescentes brasileiros, leucodermas, de origem mediterrânea. 1979. 141f. Tese ( Livre-Docência)-Faculdade de Odontologia de Bauru, Universidade de São Paulo, Bauru, 1979.

32. MARTINS, D. R. Atlas de crescimento craniofacial. São Paulo: Ed. Santos, 1998.

33. McNAMARA JR., J. A. A method of cephalometric evaluation. Am J Orthod, St. Louis, v. 86, no. 6, p. $449-469,1984$.

34. MICHIELS, L. Y. F.; TOURNE, L. P. M. Nasion true vertical: a proposed method for testing the clinical validity of cephalometric measurements applied to a new cephalometric reference line. Int J Adult Orthodon Orthognath Surg, Chicago, v. 5, no. 1, p. $43-52,1990$

35. MOORE, J. W. Variation of the sella - nasion plane and its effect on SNA and SNB. J Oral Surg, Chicago, v. 34, no. 1, p. 24-26, 1976.

36. MOORREES, C. F. A. Natural head position. In: JACOBSON, A.; CAUFIELD, P. W. Introduction to radiographic cephalometry. Philadelphia: Lea \& Febiger, 1985. cap. 8, p. 84-89.

37. MOORREES, C. F. A. Natural head position: a revival. Am J Orthod Dentofacial Orthop, St. Louis, v. 105, no. 5, p. 512-513, 1994.

38. MOORREES, C. F. A.; KEAN, M. R. Natural head position: a basic consideration in the interpretation of cephalometric radiographs. Am J Phys Anthropol, New York, v. 16, no. 2, p. $213-234,1958$

39. OPDEBEECK, H.; BELL, W. H. The short face syndrome. Am J Orthod, St. Louis, v. 73, no. 6, p. 499- 511, 1978.

40. OZBEK, M. M.; KOKLU, A. Natural cervical inclination and craniofacial structure. Am J Orthod Dentofacial Orthop, St. Louis, v. 104 , no. 6, p. $584-591,1993$

41. PENG, L.; COOKE, M. S. Fifteen year reproducibility of natural head posture: a longitudinal study. Am J Orthod Dentofacial Orthop, St. Louis, v. 116, no. 1, p. 82 - 85, 1999.

42. PRESTON, C. B.; EVANS, W. G.; TODRES, J. I. The relationship between ortho head posture and head posture measured during walking. Am J Orthod Dentofacial Orthop, St. Louis, v. 111, no. 3, p. 283 - 287, 1997.

43. PROFFIT, W. R. Contemporary Orthodontics. 2nd ed. St. Louis: C. V. Mosby, 1993

44. PROFFIT, W. R.; WHITE, R. Surgical orthodontic treatment. St. Louis: C. V. Mosby, 1990. p. 109-111, 117-124.

45. RAJU, N. S.; PRASAD, K. G.; JAYADE, V. P. A modified approach for obtaining cephalograms in the natural head position. J Orthod, Oxford, v. 28 , no.1, p. $25-28,2001$

46. RICKETTS, R. M. New prespectives on orientation and their benefits to clinical orthodontics. Part I. Angle Orthod, Appleton, v. 45 , no. 4 , p. $238-248,1975$

47. RICKETTS, R. M.; ROTH, R. J.; CHACONAS, S. J.; SCHULHOF, R. J.; ENGEL, G. A. Orthodontic diagnosis and planning their roles in preventive and rehabilitative dentistry. Am J Orthod, St. Louis, v. 46, no. 9, p. 647-673, 1960

48. RICKETTS, R. M.; SCHULHOF, R. J.; BAGHA, L. Orientation sella - nasion or Frankfort horizontal. Am J Orthod, St. Louis, v. 69, no. 6, p. 648-654, 1976

49. RINO NETO, J.; FREIRE- MAIA, B. V. A.; PAIVA, J. B. Método de registro da posição natural para obtenção da radiografia cefalométrica lateral: considerações e importância do método no diagnóstico ortodôntico- cirúrgico. R Dental Press Ortodon Ortop Facial, Maringá, v. 8, no. 3, p. 61-71, 2003

50. RINO NETO, J.; PAIVA, J. B.; FREIRE-MAIA, B. A. V.; MIASIRO JÚNIOR, H.; ATTIZZANI, M. F.; CRIVELLO JÚNIOR, O. Avaliação da reprodutibilidade da posição natural da cabeça: estudo radiográfico. Ortodontia, São Paulo, v. 35, no. 4, p. 55-68, 2002. 
51. ROCABADO, M. Biomechanical relationship of the cranial cervical and hyoid regions. Phys Ther, Alexandria, v. 1, no. 2, p. 62- 66, 1983.

52. SALZMANN, J. A. Limitations of roentgenographic cephalometrics. Am J Orthod, St. Louis, v. 50, no. 3, p. 169-88, 1964.

53. SANDHAM, A.; ORTH, D. Repeatability of head posture recordings from lateral cephalometric radiographs. Br J Orthod, Oxford, v. 15, no. 3, p. 157- 162, 1988

54. SARHAN, O. A. Rotational effects of S-N on the dentoskeletal pattern within the range of normal. Angle Orthod, Appleton, v. 59 , no. 1, p. $43-49,1989$.

55. SASSOUNI, V. A roentgenographic cephalometric analysis of cephalo- facial- dental relationships. Am J Orthod, St. Louis, v. 41, no. 6, p. 735-764, 1955

56. SHOWFETY, K. J. et al. A simple method for taking natural head position cephalograms. Am J Orthod, St. Louis, v. 83, no. 6, p. 495 - 500, 1983.

57. SIERSBAEK - NIELSEN, S.; SOLOW, B. Intra and inter- examiner variability in head posture recorded by dental auxiliaries. Am J Orthod Dentofacial Orthop, St. Louis, v. 82, no.1, p. 50-57, 1982.

58. SOLOW, B. et al. Airway adequacy, head posture and craniofacial morphology. Am J Orthod, St. Louis, v. 86, no. 4, p. 214 - 223, 1984.

59. SOLOW, B.; KREIBORG, S. Soft tissue stretching: a possible control factor in craniofacial morphogenesis. Scand J Dent Res, Copenhagen, v. 85, no. 5, p. 505-507, 1977

60. SOLOW, B.; TALLGREN, A. Natural head position in standing subjects. Acta Odontol Scand, Stockholm, v. 29, no. 5, p. 591- 607, 1971.

61. SOLOW, B.; TALLGREN, A. Head posture and craniofacial morphology. Am J Phys Anthropol, New York, v. 44, no. 3, p. $417-436,1976$.
62. SOLOW, B.; TALLGREN, A. Dentoalveolar morphology in relation to craniocervical posture. Angle Orthod, Appleton, v. 47 no. 3, p. 157-164, 1977.

63. USUMEZ, S.; ORHAN, M. Inclinometer method for recording and transferring natural head position in cephalometrics. Am J Orthod Dentofacial Orthop, St. Louis, v. 120, no. 6 p. 664- 670, 2001

64. VARGAS NETO, J.; PINZAN, A.; HENRIQUES, J. F. C.; FREITAS M. R.; JANSON, G. R. P.; ALMEIDA, R. R. Avaliação comparativa entre a linha Sela - Násio e o plano horizontal de Frankfurt como parâmetros para o diagnóstico das posições ântero - posteriores e vertical das bases ósseas, em jovens brasileiros, leucodermas, com más oclusões de Classe I e || de Angle. R Dental Press Ortodon Ortop Facial, Maringá, v. 4, n. 2, p. 13-22, 1999.

65. VIAZIS, A. D. A cephalometric analysis based on natural head position. J Clin Orthod, Boulder, v. 25, no. 3, p. 172-181, 1991.

66. VIAZIS, A. D. Anteroposterior assessment of the maxilla and mandible based on the true horizontal. J Clin Orthod, Boulder, v. 26 , no. 6, p. 673-680, 1992.

67. VIAZIS, A. D. Atlas of orthodontics: principles and clinical applications. Philadelphia: Saunders, 1993, cap. 1, p. 74

68. VIAZIS, A. D. Posição natural da cabeça. In: Atlas de Ortodontia: princípios e aplicações clínicas. São Paulo: Ed. Santos, 1996 cap. 1, p. 41-43.

69. VIG, P. S. et al. Experimental manipulation of head posture. Am J Orthod Dentofacial Orthop, St. Louis, v. 77, no. 3 p. 258-268, 1980.

70. WESSON, M. E. The ocular significance of abnormal head postures. Br J Orthod, Oxford, v. 21, p. 14-28, 1964

71. WYLIE, G. A.; FISH, L. C.; EPKER, B. N. Cephalometrics: a comparison of five analysis currently used in the diagnosis of dentofacial deformities. Int J Adult Orthod Orthog Surg, Lombard, v. 1, no. 2, p. 15- 36, 1987
Endereço para correspondência

Sérgio Carmelo Tôrres

Rua Teodoro Sampaio, 352, cj. 173

CEP 05.406-000 São Paulo/SP

E-mail: sctorres@iconet.com.br 